\title{
In Silico Exploration for Maximal Charge Transport in Organized Tetrabenzoacenes through Pitch and Roll Displacements
}

Alfy Benny, ${ }^{\dagger[a]}$ Devika Sasikumar ${ }^{\dagger[a]}$ and Mahesh Hariharan ${ }^{*[a]}$

${ }^{\dagger}$ These authors contributed equally to this work

School of Chemistry, Indian Institute of Science Education and Research Thiruvananthapuram (IISER-TVM), Maruthamala P. O., Vithura, Thiruvananthapuram 695551, Kerala, India.

\section{Supporting Information}

\section{Contents}

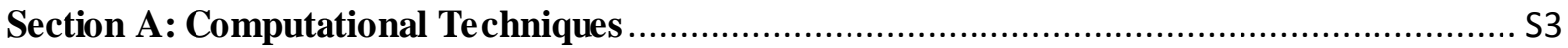

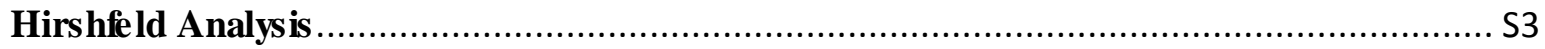

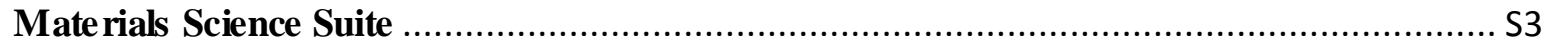

Symmetry Adapted Perturbation Theory (SAPT) ......................................................... 44

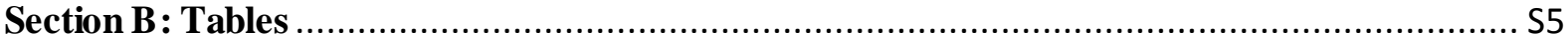

Table S1: Hirshfeld analysis providing the percentage of intermolecular interactions in NTP,

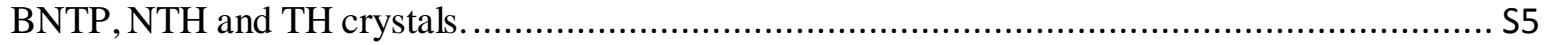

Table S2: Shows the interaction energies in selected dimers of NTP, BNTP, NTH and TH analogs determined by SAPT(0) analysis

Table S3: Shows the relation between interplanar distance to the electrostatic and exchange interaction energies in the crystalline dimers of TBA analogs.................................................. S5

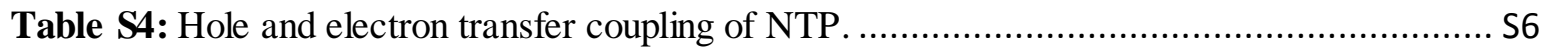

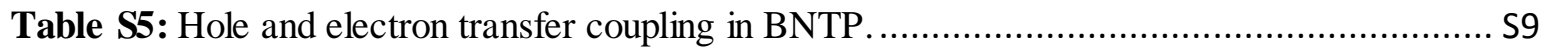

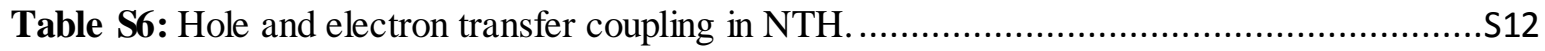

Table S7: Hole and electron transfer coupling in TH.....................................................

Table S8: Charge mobility of TBA derivatives............................................................ 18

Table S9: Anisotropic dependence of hole/electron mobility for NTP ................................S18

Table S10: Anisotropic dependence of hole/electron mobility for BNTP ...............................S19

Table S11: Anisotropic dependence of hole/electron mobility for NTH..................................S20

Table S12: Anisotropic dependence of hole/electron mobility for TH. ..................................S22

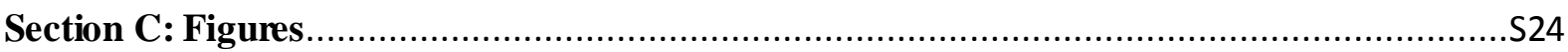


Figure S1 XRD diagram showing variation in the modes of interaction for a) NTP, b) BNTP, c)

NTH and TH that has directed the crystalline packing arrangement.

Figure S2 Crystal packing of a) NTP, b) BNTP, c) NTH and d) TH....................................S24

Figure S3 Hirshfeld fingerprint plots of a) NTP, b) BNTP, c) NTH and d) TH......................S25

Figure $\mathbf{S 4} \mathrm{N} \cdots \mathrm{C}$ interactions present in a) NTP, b) BNTP and c) NTH crystalline dimers.........S25

Figure S5 Schematic representation of the calculation of reorganization energy.......................S25

Figure S6 Shows the cofacial dimers $\left(\mathrm{D}_{0}\right)$ of the TBA derivatives at an interplanar spacing of 3.78 $\AA$ a) NTP, b) BNTP, c) NTH and d) TH.

Figure S7 Charge transfer parameters of cofacial dimers $\left(D_{0}\right)$ at an interplanar spacing of $3.78 \AA$. $\lambda_{\mathrm{h}}$ and $\lambda_{\mathrm{e}}$ are hole and electron reorganization energies while $\mathrm{V}_{\mathrm{h}}$ and $\mathrm{V}_{\mathrm{e}}$ are hole and electron coupling values respectively.

Figure S8 Representative figure showing the long $\left(\Delta_{\mathrm{L}}\right)$ and short axis $\left(\Delta_{\mathrm{S}}\right)$ displacements in NTP dimer.

Figure S9 Graphitic AA' and AB orientations of representative NTP dimer.

Figure S10 Representative figure showing the principle axis a and plane of interest ab for the anisotropic calculations in NTP cluster.

Section D: References 


\section{Section A: Computational Techniques}

\section{Hirshfeld Analysis}

The hirshfeld surface ${ }^{1-3}$ (HS) was generated using Crystal Explorer version 3.1 to quantitatively estimate the different intermolecular interactions present in the crystal structures of NTP, BNTP, NTH and TH. The HS was constructed from a set of points in the three-dimensional space which satisfy a value of 0.5 in the ratio between the electron densities of pro molecule and pro crystal. Further, the exploration of intermolecular contacts is obtained by mapping the normalized contact distances $\left(\mathrm{d}_{\text {norm }}\right)$, which is a function of the closest distances from a point on the surface to the nearest nucleus that are present inside $\left(d_{i}\right)$ and outside $\left(d_{e}\right)$ to the surface respectively. $d_{\text {norm }}$ also depends on the van der Waals radii of the nuclei present in the interior $\left(r_{i}\right)$ and exterior $\left(r_{e}\right)$ of the HS. Finally, the 2D fingerprint plot was generated from the Hirshfeld surface by plotting the fraction of points on the surface as the function of $d_{i}$ and $d_{e}$ which provide a visual summary of intermolecular contacts within the crystal.

$$
d_{\text {norm }}=\frac{d_{i}-r_{i}}{r_{i}}+\frac{d_{e}-r_{e}}{r_{e}}
$$

\section{Materials Science Suite}

The Jaguar ${ }^{4}$ DFT module from Schrodinger Materials Science Suite $2016-4^{5}$ was used for evaluating the charge transport. All the calculations were performed at B3LYP/LAC3VP** level of theory on the geometries taken from the crystal structure. The charge transfer rate, $\mathrm{k}_{\mathrm{i}}$, which represents the rate of hole/electron hopping to the similar $\mathrm{i}^{\text {th }}$ neighbor ${ }^{6}$ was obtained from the Marcus-Hush equation: $:^{7-9}$

$$
k_{i}=\frac{V^{2}}{\hbar}\left(\frac{\pi}{\lambda k_{B} T}\right)^{1 / 2} e^{\frac{-\lambda}{4 k_{B} T}}
$$

where $\mathrm{V}$ is electronic coupling between the chromophores, $\lambda$ is the reorganization energy for the respective charge carriers, $\mathrm{k}_{\mathrm{B}}$ is the Boltzmann constant and $\mathrm{T}$ is the temperature $(300 \mathrm{~K})$. The reorganization energies are given by the equation:

$$
\lambda_{ \pm}=\left(E_{0}^{*}-E_{0}\right)+\left(E_{ \pm}^{*}-E_{ \pm}\right)
$$

in which $\mathrm{E}_{0}$ and $\mathrm{E}_{ \pm}$are the lowest energies of neutral and cationic/anionic species respectively. For hole reorganization energy $\left(\lambda_{\mathrm{h}}\right), \mathrm{E}_{0}{ }^{*}$ is the energy of a neutral molecule with cation geometry while for the electron reorganization energy $\left(\lambda_{\mathrm{e}}\right), \mathrm{E}_{0}{ }^{*}$ corresponds to the energy of the neutral molecule with anion geometry (Figure S1).

The diffusion coefficient (D) was calculated from the hopping rates $\left(\mathrm{k}_{\mathrm{i}}\right)$ as follows:

$$
D=\frac{1}{2 N} \sum_{i} r^{2}{ }_{i} k_{i} P_{i}
$$

where $\mathrm{N}$ is the dimensionality of distance $(\mathrm{N}=3), \mathrm{P}_{\mathrm{i}}$ is the probability of hole/electron transfer to the $\mathrm{i}^{\text {th }}$ neighbor which is given by:

$$
P_{i}=\frac{k_{i}}{\sum_{i} k_{i}}
$$

The drift mobility of hopping was calculated from the Einstein relationship which is: 


$$
\mu=\frac{e}{k_{B} T} D
$$

Anisotropic mobility of the crystals was calculated by the following equation

$$
\mu_{\phi}=\frac{e}{2 k_{B} T} \sum_{i} r^{2} k_{i} P_{i}\left(\cos \gamma_{i}\right)^{2}\left(\cos \left(\theta_{i}-\phi\right)\right)^{2}
$$

in which $\gamma_{\mathrm{i}}$ is the angle between the charge hopping pathway and plane of interest. $\theta-\phi$ is the angle between the hopping pathway and the conduction channel. The calculations were performed on the molecular clusters obtained from the crystal structures of NTP, BNTP, NTH and TH derivatives.

\section{Symmetry Adapted Perturbation Theory (SAPT)}

Non-covalent interaction energies of dimer molecules of the TBA derivatives analysed herein were quantified using the SAPT technique which is a form of energy decomposition analysis (EDA). The energy parameters contributing to the total interaction energy which included electrostatics, exchange repulsion, induction, and dispersion interactions were obtained from a second order perturbation expansion. This includes first order electrostatics and exchange energy parts and second order dispersion, induction and their exchange counter parts as the perturbation terms.

$$
E_{\text {int }}^{S A P T(0)}=E_{\text {elc }}^{(1)}+E_{\text {ex }}^{(1)}+E_{\text {ind }}^{(2)}+E_{\text {ind-ex }}^{(2)}+E_{\text {dis }}^{(2)}+E_{\text {dis }-e x}^{(2)}
$$

The SAPT module of Psi4 code $^{10}$ was used at jun-cc-pvdz basis set. Distinguishable face-to-face

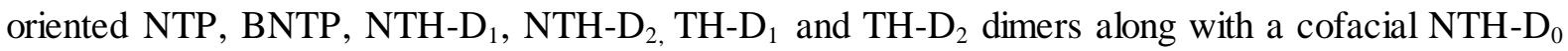
dimer were used for the SAPT calculation.

\section{Gaussian Energy Calculations}

Energy calculations of TBA monomers were performed by employing the B3LYP functional and 6$311 \mathrm{G}^{* *}$ basis set at DFT level of theory using Gaussian 09 suite. ${ }^{11}$ The frontier molecular orbitals of NTP, BNTP, NTH and TH monomers were then procured from the generated cube files of energy calculations. 


\section{Section B: Tables}

Table S1: Hirshfeld analysis providing the percentage of intermolecular interactions in NTP, BNTP, NTH and TH crystals.

\begin{tabular}{cccccccccccc}
\hline Inte raction & $\begin{array}{c}\% \\
\mathrm{C} \cdots \mathrm{C}\end{array}$ & $\begin{array}{c}\% \\
\mathrm{C} \cdots \mathrm{H}\end{array}$ & $\begin{array}{c}\% \\
\mathrm{H} \cdots \mathrm{H}\end{array}$ & $\begin{array}{c}\% \\
\mathrm{~N} \cdots \mathrm{H}\end{array}$ & $\begin{array}{c}\% \\
\mathrm{~N} \cdots \mathrm{N}\end{array}$ & $\begin{array}{c}\% \\
\mathrm{~N} \cdots \mathrm{C}\end{array}$ & $\begin{array}{c}\% \\
\mathrm{~N} \cdots \mathrm{Br}\end{array}$ & $\begin{array}{c}\% \\
\mathrm{C} \cdots \mathrm{Br}\end{array}$ & $\begin{array}{c}\% \\
\mathrm{H} \cdots \mathrm{Br}\end{array}$ & $\begin{array}{c}\% \\
\mathrm{Br} \cdots \mathrm{Br}\end{array}$ & $\begin{array}{c}\rho=[(\% \\
\mathrm{C} \cdots \mathrm{H} /\end{array}$ \\
\hline NTP & 22.9 & 14.5 & 49.4 & 7.4 & 0.6 & 5.1 & -- & -- & - & -- & 0.633 \\
BNTP & 11.2 & 25.5 & 14.0 & 1.3 & 0.1 & 8.0 & 2.4 & 1.2 & 32.3 & 4.1 & 0.709 \\
NTH & 23.2 & 18.1 & 47.7 & 6.0 & 1.3 & 3.7 & -- & -- & -- & -- & 0.780 \\
TH & 28.9 & 16.9 & 54.2 & -- & -- & -- & -- & -- & -- & -- & 0.584 \\
\hline \multicolumn{7}{c}{ total percentage of intermolecular contacts is $100 \%$ in NTP, BNTP, NTH and TH. $\rho=$} \\
{$[(\% \mathrm{C} \cdots \mathrm{H}) /(\% \mathrm{C} \cdots \mathrm{C})]$. All fall under $\beta$ packing motif $[\beta(0.46<\rho<1.0)]$} \\
\hline
\end{tabular}

Table S2: Shows the interaction energies in selected dimers of NTP, BNTP, NTH and $\mathrm{TH}$ analogs determined by SAPT( 0$)$ analysis.

\begin{tabular}{cccccc}
\hline Dimer & $\begin{array}{c}\text { Electrostatics } \\
(\mathbf{k J} / \mathbf{m o l})\end{array}$ & $\begin{array}{c}\text { Exchange } \\
(\mathbf{k J} / \mathbf{m o l})\end{array}$ & $\begin{array}{c}\text { Induction } \\
(\mathbf{k J} / \mathbf{m o l})\end{array}$ & $\begin{array}{c}\text { Dispersion } \\
(\mathbf{k J} / \mathbf{m o l})\end{array}$ & $\begin{array}{c}\text { SAPT0 } \\
(\mathbf{k J} / \mathbf{m o l})\end{array}$ \\
\hline NTP & -75.79 & 198.22 & -17.85 & -290.64 & -186.06 \\
BNTP & -83.99 & 206 & -21.57 & -304.93 & -204.48 \\
NTH-D & -81.32 & 200.60 & -17.65 & -317.87 & -216.24 \\
NTH-D & -60.04 & 165.88 & -15.36 & -301.50 & -211.02 \\
TH-D & -57.57 & 166.84 & -16.30 & -303.29 & -210.32 \\
TH-D $_{2}$ & -54.98 & 165.68 & -16.32 & -305.59 & -211.22 \\
\hline
\end{tabular}

Table S3: Shows the relation between interplanar distance to the electrostatic and exchange interaction energies in the crystalline dimers of TBA analogs.

\begin{tabular}{lcll}
\hline Dimer & $\begin{array}{c}\text { Interplanar spacing }\left(\mathbf{d}_{\mathbf{s}}\right) \\
(\AA)\end{array}$ & $\begin{array}{c}\text { Electrostatics } \\
(\mathbf{k J} / \mathbf{m o l})\end{array}$ & $\begin{array}{c}\text { Exchange } \\
(\mathbf{k J} / \mathbf{m o l})\end{array}$ \\
\hline $\mathrm{NTP}$ & 3.39 & -75.79 & 198.22 \\
$\mathrm{BNTP}$ & 3.30 & -83.99 & 206 \\
$\mathrm{NTH}\left(\mathrm{D}_{1}\right)$ & 3.40 & -81.32 & 200.60 \\
$\operatorname{NTH}\left(\mathrm{D}_{2}\right)$ & 3.51 & -60.04 & 165.88
\end{tabular}




\begin{tabular}{llll}
$\mathrm{TH}\left(\mathrm{D}_{1}\right)$ & 3.54 & -57.57 & 166.84 \\
$\mathrm{TH}\left(\mathrm{D}_{2}\right)$ & 3.55 & -54.98 & 165.68 \\
\hline
\end{tabular}

Table S4: Hole and electron transfer coupling of NTP.

\begin{tabular}{|c|c|c|c|}
\hline$\Delta_{\mathrm{L}}$ & $\Delta_{\mathrm{s}}$ & $\begin{array}{c}\text { Hole transfer } \\
\text { coupling }\left(V_{h}\right) \\
(e V)\end{array}$ & $\begin{array}{c}\text { Electron transfer } \\
\text { coupling }\left(V_{\mathrm{e}}\right) \\
(\mathrm{eV})\end{array}$ \\
\hline 4.73 & 2.0 & 0.02715 & 0.06164 \\
\hline 4.73 & 1.8 & $7.55236 \times 10^{-4}$ & 0.05802 \\
\hline 4.73 & 1.6 & 0.02663 & 0.05411 \\
\hline 4.73 & 1.4 & 0.05389 & 0.05083 \\
\hline 4.73 & 1.2 & 0.08083 & 0.04778 \\
\hline 4.73 & 1.0 & 0.10705 & 0.04708 \\
\hline 4.73 & 0.8 & 0.12964 & 0.04844 \\
\hline 4.73 & 0.6 & 0.14722 & 0.05028 \\
\hline 4.73 & 0.4 & 0.15817 & 0.05211 \\
\hline 4.73 & 0.2 & 0.16647 & 0.0493 \\
\hline 4.73 & 0 & 0.16413 & 0.05267 \\
\hline 4.1 & 2.0 & 0.03301 & 0.1809 \\
\hline 4.1 & 1.8 & 0.03977 & 0.1767 \\
\hline 4.1 & 1.6 & 0.04446 & 0.1713 \\
\hline 4.1 & 1.4 & 0.05273 & 0.16362 \\
\hline 4.1 & 1.2 & 0.05686 & 0.15732 \\
\hline 4.1 & 1.0 & 0.05836 & 0.14923 \\
\hline 4.1 & 0.8 & 0.05795 & 0.14177 \\
\hline 4.1 & 0.6 & 0.05648 & 0.13469 \\
\hline 4.1 & 0.4 & 0.05358 & 0.12955 \\
\hline 4.1 & 0.2 & 0.05029 & 0.12473 \\
\hline 4.1 & 0 & 0.0466 & 0.12409 \\
\hline 3.56 & 2.0 & 0.06055 & 0.17618 \\
\hline 3.56 & 1.8 & 0.05253 & 0.16679 \\
\hline 3.56 & 1.6 & 0.04105 & 0.15582 \\
\hline 3.56 & 1.4 & 0.02575 & 0.14167 \\
\hline 3.56 & 1.2 & 0.01185 & 0.12636 \\
\hline 3.56 & 1.0 & 0.00315 & 0.11262 \\
\hline
\end{tabular}




\begin{tabular}{|c|c|c|c|}
\hline 3.56 & 0.8 & 0.02314 & 0.09529 \\
\hline 3.56 & 0.6 & 0.04055 & 0.08054 \\
\hline 3.56 & 0.4 & 0.05282 & 0.06548 \\
\hline 3.56 & 0.2 & 0.0591 & 0.06395 \\
\hline 3.56 & 0 & 0.06836 & 0.05749 \\
\hline 2.82 & 2 & 0.02026 & $3.49322 \times 10^{-4}$ \\
\hline 2.82 & 1.8 & $2.53278 \times 10^{-4}$ & 0.01777 \\
\hline 2.82 & 1.6 & 0.02661 & 0.04455 \\
\hline 2.82 & 1.4 & 0.04721 & 0.0666 \\
\hline 2.82 & 1.2 & 0.07476 & 0.09041 \\
\hline 2.82 & 1 & 0.09697 & 0.11111 \\
\hline 2.82 & 0.8 & 0.11643 & 0.13069 \\
\hline 2.82 & 0.6 & 0.13519 & 0.15094 \\
\hline 2.82 & 0.4 & 0.14668 & 0.16276 \\
\hline 2.82 & 0.2 & 0.1495 & 0.17062 \\
\hline 2.82 & 0 & 0.15278 & 0.16746 \\
\hline 1.14 & 2 & 0.02447 & 0.13146 \\
\hline 1.14 & 1.8 & 0.04333 & 0.14862 \\
\hline 1.14 & 1.6 & 0.06291 & 0.16818 \\
\hline 1.14 & 1.4 & 0.08016 & 0.18764 \\
\hline 1.14 & 1.2 & 0.09425 & 0.20996 \\
\hline 1.14 & 1 & 0.10009 & 0.2274 \\
\hline 1.14 & 0.8 & 0.10838 & 0.24604 \\
\hline 1.14 & 0.6 & 0.11402 & 0.2609 \\
\hline 1.14 & 0.4 & 0.11661 & 0.27119 \\
\hline 1.14 & 0.2 & 0.11594 & 0.27555 \\
\hline 1.14 & 0 & 0.1117 & 0.27318 \\
\hline 1.69 & 2 & 0.09095 & 0.16317 \\
\hline 1.69 & 1.8 & 0.08733 & 0.1694 \\
\hline 1.69 & 1.6 & 0.07932 & 0.1854 \\
\hline 1.69 & 1.4 & 0.06602 & 0.17957 \\
\hline 1.69 & 1.2 & 0.05205 & 0.18314 \\
\hline 1.69 & 1 & 0.03413 & 0.18926 \\
\hline 1.69 & 0.8 & 0.00905 & 0.19108 \\
\hline 1.69 & 0.6 & 0.00893 & 0.19663 \\
\hline
\end{tabular}




\begin{tabular}{|c|c|c|c|}
\hline 1.69 & 0.4 & 0.02187 & 0.19956 \\
\hline 1.69 & 0.2 & 0.0355 & 0.20707 \\
\hline 1.69 & 0 & 0.0409 & 0.20017 \\
\hline 1.02 & 2 & 0.15581 & 0.05321 \\
\hline 1.02 & 1.8 & 0.1204 & 0.02619 \\
\hline 1.02 & 1.6 & 0.07579 & 0.0113 \\
\hline 1.02 & 1.4 & 0.02662 & 0.01048 \\
\hline 1.02 & 1.2 & 0.03054 & 0.03064 \\
\hline 1.02 & 1 & 0.08627 & 0.04654 \\
\hline 1.02 & 0.8 & 0.13953 & 0.0743 \\
\hline 1.02 & 0.6 & 0.18667 & 0.0913 \\
\hline 1.02 & 0.4 & 0.23112 & 0.10583 \\
\hline 1.02 & 0.2 & 0.26224 & 0.11889 \\
\hline 1.02 & 0 & 0.2765 & 0.12402 \\
\hline 0.49 & 2 & 0.15894 & 0.08284 \\
\hline 0.49 & 1.8 & 0.09599 & 0.11445 \\
\hline 0.49 & 1.6 & 0.02319 & 0.16573 \\
\hline 0.49 & 1.4 & 0.05329 & 0.20429 \\
\hline 0.49 & 1.2 & 0.1372 & 0.24665 \\
\hline 0.49 & 1 & 0.21789 & 0.28906 \\
\hline 0.49 & 0.8 & 0.29016 & 0.32732 \\
\hline 0.49 & 0.6 & 0.38568 & 0.35806 \\
\hline 0.49 & 0.4 & 0.39831 & 0.37923 \\
\hline 0.49 & 0.2 & 0.42884 & 0.33852 \\
\hline 0.49 & 0 & 0.41884 & 0.39636 \\
\hline 0 & 2 & 0.182 & 0.13467 \\
\hline 0 & 1.8 & 0.11766 & 0.17137 \\
\hline 0 & 1.6 & 0.02656 & 0.2198 \\
\hline 0 & 1.4 & 0.06574 & 0.27222 \\
\hline 0 & 1.2 & 0.16232 & 0.32852 \\
\hline 0 & 1 & 0.25815 & 0.38488 \\
\hline 0 & 0.8 & 0.34568 & 0.44113 \\
\hline 0 & 0.6 & 0.41884 & 0.48116 \\
\hline 0 & 0.4 & 0.4709 & 0.51019 \\
\hline 0 & 0.2 & 0.50005 & 0.52782 \\
\hline
\end{tabular}




$\begin{array}{llll}0 & 0 & 0.50835 & 0.53436\end{array}$

Table S5: Hole and electron transfer coupling in BNTP.

\begin{tabular}{|c|c|c|c|}
\hline$\Delta_{\mathrm{L}}$ & $\Delta_{\mathrm{s}}$ & $\begin{array}{l}\text { Hole transfer } \\
\text { coupling }\left(V_{h}\right) \\
(e V)\end{array}$ & $\begin{array}{c}\text { Electron transfer } \\
\text { coupling }\left(V_{e}\right) \\
(e V)\end{array}$ \\
\hline 4.96 & 2.0 & 0.05069 & 0.14256 \\
\hline 4.96 & 1.8 & 0.0384 & 0.13599 \\
\hline 4.96 & 1.6 & 0.11419 & 0.1271 \\
\hline 4.96 & 1.4 & 0.14784 & 0.11748 \\
\hline 4.96 & 1.2 & 0.21361 & 0.12403 \\
\hline 4.96 & 1.0 & 0.26888 & 0.1067 \\
\hline 4.96 & 0.8 & 0.2949 & 0.10418 \\
\hline 4.96 & 0.6 & 0.29751 & 0.10417 \\
\hline 4.96 & 0.4 & 0.38998 & 0.0278 \\
\hline 4.96 & 0.2 & 0.32129 & 0.06442 \\
\hline 4.96 & 0 & 0.22543 & 0.10481 \\
\hline 4.31 & 2.0 & 0.11907 & 0.145 \\
\hline 4.31 & 1.8 & 0.01738 & 0.18794 \\
\hline 4.31 & 1.6 & 0.16421 & 0.23083 \\
\hline 4.31 & 1.4 & 0.28977 & 0.26952 \\
\hline 4.31 & 1.2 & 0.40025 & 0.30426 \\
\hline 4.31 & 1.0 & 0.49345 & 0.33731 \\
\hline 4.31 & 0.8 & 0.54537 & 0.34407 \\
\hline 4.31 & 0.6 & 0.57021 & 0.36533 \\
\hline 4.31 & 0.4 & 0.43788 & 0.15946 \\
\hline 4.31 & 0.2 & 0.35763 & 0.19832 \\
\hline 4.31 & 0 & 0.24764 & 0.25839 \\
\hline 3.67 & 2.0 & 0.11289 & 0.3179 \\
\hline 3.67 & 1.8 & 0.04482 & 0.37763 \\
\hline 3.67 & 1.6 & 0.20842 & 0.44844 \\
\hline 3.67 & 1.4 & 0.35196 & 0.51061 \\
\hline 3.67 & 1.2 & 0.47332 & 0.56235 \\
\hline 3.67 & 1.0 & 0.57206 & 0.59978 \\
\hline 3.67 & 0.8 & 0.63344 & 0.62533 \\
\hline 3.67 & 0.6 & 0.65388 & 0.63471 \\
\hline
\end{tabular}




\begin{tabular}{|c|c|c|c|}
\hline 3.67 & 0.4 & 0.05069 & 0.14256 \\
\hline 3.67 & 0.2 & 0.0384 & 0.13599 \\
\hline 3.67 & 0 & 0.11419 & 0.1271 \\
\hline 3.11 & 2 & 0.14784 & 0.11748 \\
\hline 3.11 & 1.8 & 0.21361 & 0.12403 \\
\hline 3.11 & 1.6 & 0.26888 & 0.1067 \\
\hline 3.11 & 1.4 & 0.2949 & 0.10418 \\
\hline 3.11 & 1.2 & 0.29751 & 0.10417 \\
\hline 3.11 & 1 & 0.38998 & 0.0278 \\
\hline 3.11 & 0.8 & 0.32129 & 0.06442 \\
\hline 3.11 & 0.6 & 0.22543 & 0.10481 \\
\hline 3.11 & 0.4 & 0.11907 & 0.145 \\
\hline 3.11 & 0.2 & 0.01738 & 0.18794 \\
\hline 3.11 & 0 & 0.16421 & 0.23083 \\
\hline 2.51 & 2 & 0.28977 & 0.26952 \\
\hline 2.51 & 1.8 & 0.40025 & 0.30426 \\
\hline 2.51 & 1.6 & 0.49345 & 0.33731 \\
\hline 2.51 & 1.4 & 0.54537 & 0.34407 \\
\hline 2.51 & 1.2 & 0.57021 & 0.36533 \\
\hline 2.51 & 1 & 0.43788 & 0.15946 \\
\hline 2.51 & 0.8 & 0.35763 & 0.19832 \\
\hline 2.51 & 0.6 & 0.24764 & 0.25839 \\
\hline 2.51 & 0.4 & 0.11289 & 0.3179 \\
\hline 2.51 & 0.2 & 0.04482 & 0.37763 \\
\hline 2.51 & 0 & 0.20842 & 0.44844 \\
\hline 1.9 & 2 & 0.35196 & 0.51061 \\
\hline 1.9 & 1.8 & 0.47332 & 0.56235 \\
\hline 1.9 & 1.6 & 0.57206 & 0.59978 \\
\hline 1.9 & 1.4 & 0.63344 & 0.62533 \\
\hline 1.9 & 1.2 & 0.65388 & 0.63471 \\
\hline 1.9 & 1 & 0.05069 & 0.14256 \\
\hline 1.9 & 0.8 & 0.0384 & 0.13599 \\
\hline 1.9 & 0.6 & 0.11419 & 0.1271 \\
\hline 1.9 & 0.4 & 0.14784 & 0.11748 \\
\hline 1.9 & 0.2 & 0.21361 & 0.12403 \\
\hline
\end{tabular}




\begin{tabular}{|c|c|c|c|}
\hline 1.9 & 0 & 0.26888 & 0.1067 \\
\hline 1.4 & 2 & 0.2949 & 0.10418 \\
\hline 1.4 & 1.8 & 0.29751 & 0.10417 \\
\hline 1.4 & 1.6 & 0.38998 & 0.0278 \\
\hline 1.4 & 1.4 & 0.32129 & 0.06442 \\
\hline 1.4 & 1.2 & 0.22543 & 0.10481 \\
\hline 1.4 & 1 & 0.11907 & 0.145 \\
\hline 1.4 & 0.8 & 0.01738 & 0.18794 \\
\hline 1.4 & 0.6 & 0.16421 & 0.23083 \\
\hline 1.4 & 0.4 & 0.28977 & 0.26952 \\
\hline 1.4 & 0.2 & 0.40025 & 0.30426 \\
\hline 1.4 & 0 & 0.49345 & 0.33731 \\
\hline 0.75 & 2 & 0.54537 & 0.34407 \\
\hline 0.75 & 1.8 & 0.57021 & 0.36533 \\
\hline 0.75 & 1.6 & 0.43788 & 0.15946 \\
\hline 0.75 & 1.4 & 0.35763 & 0.19832 \\
\hline 0.75 & 1.2 & 0.24764 & 0.25839 \\
\hline 0.75 & 1 & 0.11289 & 0.3179 \\
\hline 0.75 & 0.8 & 0.04482 & 0.37763 \\
\hline 0.75 & 0.6 & 0.20842 & 0.44844 \\
\hline 0.75 & 0.4 & 0.35196 & 0.51061 \\
\hline 0.75 & 0.2 & 0.47332 & 0.56235 \\
\hline 0.75 & 0 & 0.57206 & 0.59978 \\
\hline 0 & 2 & 0.63344 & 0.62533 \\
\hline 0 & 1.8 & 0.65388 & 0.63471 \\
\hline 0 & 1.6 & 0.05069 & 0.14256 \\
\hline 0 & 1.4 & 0.0384 & 0.13599 \\
\hline 0 & 1.2 & 0.11419 & 0.1271 \\
\hline 0 & 1 & 0.14784 & 0.11748 \\
\hline 0 & 0.8 & 0.21361 & 0.12403 \\
\hline 0 & 0.6 & 0.26888 & 0.1067 \\
\hline 0 & 0.4 & 0.2949 & 0.10418 \\
\hline 0 & 0.2 & 0.29751 & 0.10417 \\
\hline 0 & 0 & 0.38998 & 0.0278 \\
\hline
\end{tabular}


Table S6: Hole and electron transfer coupling in NTH.

\begin{tabular}{|c|c|c|c|}
\hline$\Delta_{\mathrm{L}}$ & $\Delta_{\mathrm{s}}$ & $\begin{array}{l}\text { Hole transfer } \\
\text { coupling }\left(V_{h}\right) \\
(e V)\end{array}$ & $\begin{array}{c}\text { Electron transfer } \\
\text { coupling }\left(V_{e}\right) \\
(e V)\end{array}$ \\
\hline 4.9 & 2.0 & 0.10877 & 0.12318 \\
\hline 4.9 & 1.8 & 0.15282 & 0.15714 \\
\hline 4.9 & 1.6 & 0.20671 & 0.19883 \\
\hline 4.9 & 1.4 & 0.26317 & 0.24787 \\
\hline 4.9 & 1.2 & 0.31134 & 0.29268 \\
\hline 4.9 & 1.0 & 0.35932 & 0.33926 \\
\hline 4.9 & 0.8 & 0.40655 & 0.3931 \\
\hline 4.9 & 0.6 & 0.44325 & 0.43556 \\
\hline 4.9 & 0.4 & 0.46609 & 0.46137 \\
\hline 4.9 & 0.2 & 0.47769 & 0.47792 \\
\hline 4.9 & 0 & 0.48015 & 0.48564 \\
\hline 4.13 & 2.0 & 0.06011 & 0.08269 \\
\hline 4.13 & 1.8 & 0.10557 & 0.11952 \\
\hline 4.13 & 1.6 & 0.14993 & 0.16143 \\
\hline 4.13 & 1.4 & 0.20356 & 0.20767 \\
\hline 4.13 & 1.2 & 0.25024 & 0.24938 \\
\hline 4.13 & 1.0 & 0.30614 & 0.29832 \\
\hline 4.13 & 0.8 & 0.35148 & 0.3422 \\
\hline 4.13 & 0.6 & 0.39483 & 0.3856 \\
\hline 4.13 & 0.4 & 0.42401 & 0.41363 \\
\hline 4.13 & 0.2 & 0.4438 & 0.4329 \\
\hline 4.13 & 0 & 0.45542 & 0.44531 \\
\hline 3.59 & 2.0 & 0.03248 & 0.02353 \\
\hline 3.59 & 1.8 & $1.31193 \times 10^{-4}$ & 0.00834 \\
\hline 3.59 & 1.6 & 0.03168 & 0.03493 \\
\hline 3.59 & 1.4 & 0.06686 & 0.06466 \\
\hline 3.59 & 1.2 & 0.10529 & 0.09758 \\
\hline 3.59 & 1.0 & 0.13818 & 0.11933 \\
\hline 3.59 & 0.8 & 0.18122 & 0.15803 \\
\hline 3.59 & 0.6 & 0.21943 & 0.19098 \\
\hline 3.59 & 0.4 & 0.23791 & 0.203 \\
\hline 3.59 & 0.2 & 0.25754 & 0.2168 \\
\hline
\end{tabular}




\begin{tabular}{|c|c|c|c|}
\hline 3.59 & 0 & 0.26456 & 0.22423 \\
\hline 3.01 & 2 & 0.16356 & 0.17329 \\
\hline 3.01 & 1.8 & 0.15707 & 0.16749 \\
\hline 3.01 & 1.6 & 0.15054 & 0.15664 \\
\hline 3.01 & 1.4 & 0.13993 & 0.14976 \\
\hline 3.01 & 1.2 & 0.12549 & 0.14186 \\
\hline 3.01 & 1 & 0.1087 & 0.13885 \\
\hline 3.01 & 0.8 & 0.09209 & 0.12794 \\
\hline 3.01 & 0.6 & 0.07451 & 0.1215 \\
\hline 3.01 & 0.4 & 0.06066 & 0.11683 \\
\hline 3.01 & 0.2 & 0.04779 & 0.11361 \\
\hline 3.01 & 0 & 0.03568 & 0.1117 \\
\hline 2.2 & 2 & 0.15547 & 0.1767 \\
\hline 2.2 & 1.8 & 0.17313 & 0.18978 \\
\hline 2.2 & 1.6 & 0.18645 & 0.1946 \\
\hline 2.2 & 1.4 & 0.19811 & 0.20568 \\
\hline 2.2 & 1.2 & 0.20891 & 0.21738 \\
\hline 2.2 & 1 & 0.21674 & 0.22194 \\
\hline 2.2 & 0.8 & 0.22206 & 0.23489 \\
\hline 2.2 & 0.6 & 0.22186 & 0.2449 \\
\hline 2.2 & 0.4 & 0.21944 & 0.25333 \\
\hline 2.2 & 0.2 & 0.21183 & 0.25727 \\
\hline 2.2 & 0 & 0.20426 & 0.25699 \\
\hline 1.54 & 2 & 0.02249 & 0.04599 \\
\hline 1.54 & 1.8 & 0.00126 & 0.04061 \\
\hline 1.54 & 1.6 & 0.02319 & 0.02568 \\
\hline 1.54 & 1.4 & 0.0467 & 0.00958 \\
\hline 1.54 & 1.2 & 0.07077 & 0.00975 \\
\hline 1.54 & 1 & 0.09302 & 0.02971 \\
\hline 1.54 & 0.8 & 0.11303 & 0.04986 \\
\hline 1.54 & 0.6 & 0.12907 & 0.06215 \\
\hline 1.54 & 0.4 & 0.14124 & 0.07878 \\
\hline 1.54 & 0.2 & 0.15041 & 0.09497 \\
\hline 1.54 & 0 & 0.14759 & 0.09432 \\
\hline 0.9 & 2 & 0.13985 & 0.19171 \\
\hline
\end{tabular}




\begin{tabular}{|c|c|c|c|}
\hline 0.9 & 1.8 & 0.12284 & 0.18502 \\
\hline 0.9 & 1.6 & 0.10256 & 0.17978 \\
\hline 0.9 & 1.4 & 0.08079 & 0.17006 \\
\hline 0.9 & 1.2 & 0.05757 & 0.15594 \\
\hline 0.9 & 1 & 0.03549 & 0.14387 \\
\hline 0.9 & 0.8 & 0.01535 & 0.12625 \\
\hline 0.9 & 0.6 & 0.00749 & 0.10592 \\
\hline 0.9 & 0.4 & 0.01839 & 0.09772 \\
\hline 0.9 & 0.2 & 0.03048 & 0.0834 \\
\hline 0.9 & 0 & 0.031 & 0.08303 \\
\hline 0.41 & 2 & 0.16367 & 0.21342 \\
\hline 0.41 & 1.8 & 0.15414 & 0.21584 \\
\hline 0.41 & 1.6 & 0.14052 & 0.2159 \\
\hline 0.41 & 1.4 & 0.12315 & 0.20828 \\
\hline 0.41 & 1.2 & 0.10676 & 0.1995 \\
\hline 0.41 & 1 & 0.08861 & 0.19015 \\
\hline 0.41 & 0.8 & 0.07134 & 0.1802 \\
\hline 0.41 & 0.6 & 0.05503 & 0.17028 \\
\hline 0.41 & 0.4 & 0.04141 & 0.16022 \\
\hline 0.41 & 0.2 & 0.02894 & 0.15229 \\
\hline 0.41 & 0 & 0.02256 & 0.153 \\
\hline 0 & 2 & 0.04022 & 0.05982 \\
\hline 0 & 1.8 & 0.03799 & 0.05656 \\
\hline 0 & 1.6 & 0.02968 & 0.05569 \\
\hline 0 & 1.4 & 0.03457 & 0.05269 \\
\hline 0 & 1.2 & 0.02315 & 0.05164 \\
\hline 0 & 1 & 0.01609 & 0.04397 \\
\hline 0 & 0.8 & 0.00907 & 0.04279 \\
\hline 0 & 0.6 & 0.00116 & 0.03622 \\
\hline 0 & 0.4 & 0.00645 & 0.03889 \\
\hline 0 & 0.2 & 0.01326 & 0.03633 \\
\hline 0 & 0 & 0.01944 & 0.03235 \\
\hline
\end{tabular}

Table S7: Hole and electron transfer coupling in TH.

$\begin{array}{llll}\Delta_{\mathrm{L}} & \Delta_{\mathrm{S}} & \text { Hole transfer } & \text { Electron transfer }\end{array}$




\begin{tabular}{|c|c|c|c|}
\hline & & $\begin{array}{c}\text { coupling }\left(V_{h}\right) \\
(e V)\end{array}$ & $\begin{array}{c}\text { coupling }\left(V_{e}\right) \\
(e V)\end{array}$ \\
\hline 5.07 & 2.0 & 0.00836 & 0.09403 \\
\hline 5.07 & 1.8 & 0.01671 & 0.09253 \\
\hline 5.07 & 1.6 & 0.04108 & 0.09774 \\
\hline 5.07 & 1.4 & 0.06695 & 0.09771 \\
\hline 5.07 & 1.2 & 0.09075 & 0.10326 \\
\hline 5.07 & 1.0 & 0.11591 & 0.10274 \\
\hline 5.07 & 0.8 & 0.14113 & 0.09624 \\
\hline 5.07 & 0.6 & 0.16164 & 0.09464 \\
\hline 5.07 & 0.4 & 0.17699 & 0.09272 \\
\hline 5.07 & 0.2 & 0.18382 & 0.09099 \\
\hline 5.07 & 0 & 0.18998 & 0.08796 \\
\hline 4.39 & 2.0 & 0.01889 & 0.25652 \\
\hline 4.39 & 1.8 & 0.01737 & 0.26304 \\
\hline 4.39 & 1.6 & 0.01526 & 0.26624 \\
\hline 4.39 & 1.4 & 0.01361 & 0.26534 \\
\hline 4.39 & 1.2 & 0.00727 & 0.26597 \\
\hline 4.39 & 1.0 & 0.00491 & 0.26389 \\
\hline 4.39 & 0.8 & 0.00474 & 0.25912 \\
\hline 4.39 & 0.6 & 0.00655 & 0.25224 \\
\hline 4.39 & 0.4 & 0.01105 & 0.24429 \\
\hline 4.39 & 0.2 & 0.02149 & 0.23677 \\
\hline 4.39 & 0 & 0.02866 & 0.22978 \\
\hline 3.78 & 2.0 & 0.03346 & 0.21537 \\
\hline 3.78 & 1.8 & 0.00635 & 0.21751 \\
\hline 3.78 & 1.6 & 0.02454 & 0.2168 \\
\hline 3.78 & 1.4 & 0.05655 & 0.21422 \\
\hline 3.78 & 1.2 & 0.08843 & 0.2084 \\
\hline 3.78 & 1.0 & 0.1169 & 0.19882 \\
\hline 3.78 & 0.8 & 0.14006 & 0.18663 \\
\hline 3.78 & 0.6 & 0.15034 & 0.18051 \\
\hline 3.78 & 0.4 & 0.16288 & 0.16506 \\
\hline 3.78 & 0.2 & 0.16325 & 0.1589 \\
\hline 3.78 & 0 & 0.15909 & 0.15478 \\
\hline
\end{tabular}




\begin{tabular}{|c|c|c|c|}
\hline 3.11 & 2 & 0.03599 & 0.0017 \\
\hline 3.11 & 1.8 & 0.01308 & 0.01173 \\
\hline 3.11 & 1.6 & 0.06661 & 0.02625 \\
\hline 3.11 & 1.4 & 0.12002 & 0.0437 \\
\hline 3.11 & 1.2 & 0.1738 & 0.06044 \\
\hline 3.11 & 1 & 0.22742 & 0.07835 \\
\hline 3.11 & 0.8 & 0.26514 & 0.09247 \\
\hline 3.11 & 0.6 & 0.29221 & 0.09638 \\
\hline 3.11 & 0.4 & 0.31186 & 0.11524 \\
\hline 3.11 & 0.2 & 0.31591 & 0.11842 \\
\hline 3.11 & 0 & 0.31736 & 0.1206 \\
\hline 2.47 & 2 & 0.07153 & 0.23021 \\
\hline 2.47 & 1.8 & 0.02922 & 0.24634 \\
\hline 2.47 & 1.6 & 0.02107 & 0.26346 \\
\hline 2.47 & 1.4 & 0.07537 & 0.27713 \\
\hline 2.47 & 1.2 & 0.12744 & 0.30076 \\
\hline 2.47 & 1 & 0.17999 & 0.31666 \\
\hline 2.47 & 0.8 & 0.22537 & 0.34093 \\
\hline 2.47 & 0.6 & 0.26413 & 0.35596 \\
\hline 2.47 & 0.4 & 0.29189 & 0.36469 \\
\hline 2.47 & 0.2 & 0.30118 & 0.37027 \\
\hline 2.47 & 0 & 0.31068 & 0.36969 \\
\hline 1.87 & 2 & 0.05388 & 0.26567 \\
\hline 1.87 & 1.8 & 0.0361 & 0.27793 \\
\hline 1.87 & 1.6 & 0.01543 & 0.29091 \\
\hline 1.87 & 1.4 & 0.00214 & 0.29898 \\
\hline 1.87 & 1.2 & 0.02957 & 0.31587 \\
\hline 1.87 & 1 & 0.05355 & 0.32723 \\
\hline 1.87 & 0.8 & 0.07895 & 0.33588 \\
\hline 1.87 & 0.6 & 0.10661 & 0.34562 \\
\hline 1.87 & 0.4 & 0.12406 & 0.34397 \\
\hline 1.87 & 0.2 & 0.13489 & 0.34409 \\
\hline 1.87 & 0 & 0.15176 & 0.34814 \\
\hline 1.13 & 2 & 0.00831 & 0.05007 \\
\hline 1.13 & 1.8 & 0.03609 & 0.0439 \\
\hline
\end{tabular}




\begin{tabular}{|c|c|c|c|}
\hline 1.13 & 1.6 & 0.06631 & 0.0364 \\
\hline 1.13 & 1.4 & 0.09382 & 0.03591 \\
\hline 1.13 & 1.2 & 0.12386 & 0.01543 \\
\hline 1.13 & 1 & 0.14749 & 0.00392 \\
\hline 1.13 & 0.8 & 0.16583 & 0.00764 \\
\hline 1.13 & 0.6 & 0.17594 & 0.01777 \\
\hline 1.13 & 0.4 & 0.17773 & 0.02595 \\
\hline 1.13 & 0.2 & 0.17408 & 0.03037 \\
\hline 1.13 & 0 & 0.16558 & 0.033 \\
\hline 0.61 & 2 & 0.05169 & 0.15793 \\
\hline 0.61 & 1.8 & 0.00569 & 0.17928 \\
\hline 0.61 & 1.6 & 0.06923 & 0.20614 \\
\hline 0.61 & 1.4 & 0.13352 & 0.24481 \\
\hline 0.61 & 1.2 & 0.19579 & 0.27058 \\
\hline 0.61 & 1 & 0.25401 & 0.3011 \\
\hline 0.61 & 0.8 & 0.3067 & 0.32925 \\
\hline 0.61 & 0.6 & 0.34573 & 0.35201 \\
\hline 0.61 & 0.4 & 0.36901 & 0.3678 \\
\hline 0.61 & 0.2 & 0.37059 & 0.36471 \\
\hline 0.61 & 0 & 0.3702 & 0.36882 \\
\hline 0.07 & 2 & 0.10694 & 0.26156 \\
\hline 0.07 & 1.8 & 0.04623 & 0.28986 \\
\hline 0.07 & 1.6 & 0.03804 & 0.32751 \\
\hline 0.07 & 1.4 & 0.11801 & 0.36598 \\
\hline 0.07 & 1.2 & 0.20187 & 0.40528 \\
\hline 0.07 & 1 & 0.27886 & 0.44627 \\
\hline 0.07 & 0.8 & 0.34772 & 0.48521 \\
\hline 0.07 & 0.6 & 0.4014 & 0.51716 \\
\hline 0.07 & 0.4 & 0.43704 & 0.53806 \\
\hline 0.07 & 0.2 & 0.45174 & 0.54483 \\
\hline 0.07 & 0 & 0.45788 & 0.54952 \\
\hline 0 & 2 & 0.14774 & 0.24533 \\
\hline 0 & 1.8 & 0.08906 & 0.27512 \\
\hline 0 & 1.6 & 0.01437 & 0.30861 \\
\hline 0 & 1.4 & 0.06493 & 0.34464 \\
\hline
\end{tabular}




\begin{tabular}{cccc}
0 & 1.2 & 0.14948 & 0.38424 \\
0 & 1 & 0.23003 & 0.42486 \\
0 & 0.8 & 0.30595 & 0.4665 \\
0 & 0.6 & 0.36967 & 0.50332 \\
0 & 0.4 & 0.41803 & 0.53209 \\
0 & 0.2 & 0.44696 & 0.54898 \\
0 & 0 & 0.45945 & 0.555 \\
\hline
\end{tabular}

Table S8: Charge mobility of TBA derivatives.

\begin{tabular}{ccc}
\hline Crystal & $\begin{array}{c}\mu_{\mathrm{h}} \\
\left(\mathrm{cm}^{2} \mathrm{~V}^{1} \mathrm{~s}^{-1}\right)\end{array}$ & $\begin{array}{c}\mu_{\mathrm{e}} \\
\left(\mathrm{cm}^{2} \mathrm{~V}^{-1} \mathrm{~s}^{-1}\right)\end{array}$ \\
\hline NTP & 0.29 & 1.55 \\
BNTP & 1.48 & 0.02 \\
NTH & 8.82 & 2.28 \\
TH & 5.32 & 8.11 \\
\hline
\end{tabular}

Table S9: Anisotropic dependence of hole/electron mobility for NTP.

\begin{tabular}{ccc}
\hline $\begin{array}{c}\text { Angle } \\
(\mathbf{d e g r e e})\end{array}$ & $\begin{array}{c}\text { Hole mobility } \\
\left(\mathbf{c m}^{\mathbf{2}} \mathbf{V}^{-1} \mathbf{s}^{-\mathbf{1}}\right)\end{array}$ & $\begin{array}{c}\text { Electron mobility } \\
\left(\mathbf{c m}^{\mathbf{2}} \mathbf{V}^{-1} \mathbf{s}^{-1}\right)\end{array}$ \\
\hline 0 & 0.285833 & 1.293723 \\
10 & 0.27721 & 1.254557 \\
20 & 0.252463 & 1.142183 \\
30 & 0.214577 & 0.970153 \\
40 & 0.16812 & 0.75921 \\
50 & 0.11869 & 0.534787 \\
60 & 0.07225 & 0.32394 \\
70 & 0.0344 & 0.152093 \\
70 & 0.009703 & 0.039963 \\
80 & 0.001133 & 0.001073 \\
90 & 0.009727 & 0.04011 \\
100 & 0.03445 & 0.152367 \\
110 & 0.072317 & 0.32431 \\
120 & 0.118763 & 0.53521 \\
130 & 0.168193 & 0.759633 \\
140 & 0.21464 & 0.970527 \\
150 & 0.25251 & 1.14246 \\
& &
\end{tabular}




\begin{tabular}{ccc}
160 & 0.277233 & 1.254703 \\
170 & 0.285833 & 1.293723 \\
180 & 0.277267 & 1.254817 \\
190 & 0.25257 & 1.142677 \\
200 & 0.214723 & 0.970817 \\
210 & 0.168283 & 0.759967 \\
220 & 0.118857 & 0.53554 \\
230 & 0.072397 & 0.324603 \\
240 & 0.03451 & 0.152583 \\
250 & 0.00976 & 0.040227 \\
260 & 0.001133 & 0.001073 \\
270 & 0.00967 & 0.03985 \\
280 & 0.03434 & 0.151877 \\
290 & 0.07217 & 0.323647 \\
300 & 0.118597 & 0.534453 \\
310 & 0.168027 & 0.75888 \\
320 & 0.214493 & 0.969863 \\
330 & 0.252403 & 1.141967 \\
340 & 0.277177 & 1.25444 \\
350 & 0.285833 & 1.293723 \\
360 & 0.285833 & \\
\hline & & \\
\hline & & \\
2003723 \\
\hline
\end{tabular}

Table S10: Anisotropic dependence of hole/electron mobility for BNTP.

\begin{tabular}{ccc}
\hline $\begin{array}{c}\text { Angle } \\
(\mathbf{d e g r e e})\end{array}$ & $\begin{array}{c}\text { Hole mobility } \\
\left(\mathbf{c m}^{\mathbf{2}} \mathbf{V}^{-1} \mathbf{s}^{-\mathbf{1}}\right)\end{array}$ & $\begin{array}{c}\text { Electron mobility } \\
\left(\mathbf{c m}^{\mathbf{2}} \mathbf{V}^{\mathbf{1}} \mathbf{s}^{-1}\right)\end{array}$ \\
\hline 0 & 1.458703 & 0.00996 \\
10 & 1.415247 & 0.009953 \\
20 & 1.289947 & 0.009933 \\
30 & 1.09791 & 0.009903 \\
40 & 0.862303 & 0.009867 \\
50 & 0.61154 & 0.00983 \\
60 & 0.375873 & 0.00979 \\
70 & 0.18372 & 0.00976 \\
80 & 0.058263 & 0.009743 \\
90 & 0.01463 & 0.009733
\end{tabular}




\begin{tabular}{|c|c|c|}
\hline 100 & 0.058087 & 0.00974 \\
\hline 110 & 0.183387 & 0.00976 \\
\hline 120 & 0.375423 & 0.00979 \\
\hline 130 & 0.611033 & 0.009827 \\
\hline 140 & 0.861793 & 0.009867 \\
\hline 150 & 1.097463 & 0.009903 \\
\hline 160 & 1.289613 & 0.009933 \\
\hline 170 & 1.41507 & 0.009953 \\
\hline 180 & 1.458703 & 0.00996 \\
\hline 190 & 1.415247 & 0.009953 \\
\hline 200 & 1.289947 & 0.009933 \\
\hline 210 & 1.09791 & 0.009903 \\
\hline 220 & 0.862303 & 0.009867 \\
\hline 230 & 0.61154 & 0.00983 \\
\hline 240 & 0.375873 & 0.00979 \\
\hline 250 & 0.18372 & 0.00976 \\
\hline 260 & 0.058263 & 0.009743 \\
\hline 270 & 0.01463 & 0.009733 \\
\hline 280 & 0.058087 & 0.00974 \\
\hline 290 & 0.183387 & 0.00976 \\
\hline 300 & 0.375423 & 0.00979 \\
\hline 310 & 0.611033 & 0.009827 \\
\hline 320 & 0.861793 & 0.009867 \\
\hline 330 & 1.097463 & 0.009903 \\
\hline 340 & 1.289613 & 0.009933 \\
\hline 350 & 1.41507 & 0.009953 \\
\hline 360 & 1.458703 & 0.00996 \\
\hline
\end{tabular}

Table S11: Anisotropic dependence of hole/electron mobility for NTH.

\begin{tabular}{ccc}
\hline $\begin{array}{c}\text { Angle } \\
(\text { degree })\end{array}$ & $\begin{array}{c}\text { Hole mobility } \\
\left(\mathbf{c m}^{\mathbf{2}} \mathbf{V}^{-1} \mathbf{s}^{-\mathbf{1}}\right)\end{array}$ & $\begin{array}{c}\text { Electron mobility } \\
\left(\mathbf{c m}^{\mathbf{2}} \mathbf{V}^{-1} \mathbf{s}^{-1}\right)\end{array}$ \\
\hline 0 & 8.823947 & 2.27558 \\
10 & 8.557873 & 2.206963 \\
20 & 7.79174 & 2.009387 \\
30 & 6.61796 & 1.706683
\end{tabular}




\begin{tabular}{|c|c|c|}
\hline 40 & 5.178107 & 1.335363 \\
\hline 50 & 3.645843 & 0.940213 \\
\hline 60 & 2.20599 & 0.568893 \\
\hline 70 & 1.03221 & 0.266193 \\
\hline 80 & 0.26608 & 0.068617 \\
\hline 90 & $5.44 \times 10^{-6}$ & $1.86 \mathrm{E}-07$ \\
\hline 100 & 0.26608 & 0.068617 \\
\hline 110 & 1.03221 & 0.266193 \\
\hline 120 & 2.20599 & 0.568897 \\
\hline 130 & 3.645847 & 0.940213 \\
\hline 140 & 5.178107 & 1.335363 \\
\hline 150 & 6.617963 & 1.706683 \\
\hline 160 & 7.791743 & 2.009387 \\
\hline 170 & 8.557873 & 2.206963 \\
\hline 180 & 8.823947 & 2.27558 \\
\hline 190 & 8.557873 & 2.206963 \\
\hline 200 & 7.79174 & 2.009387 \\
\hline 210 & 6.61796 & 1.706683 \\
\hline 220 & 5.178107 & 1.335363 \\
\hline 230 & 3.645843 & 0.940213 \\
\hline 240 & 2.20599 & 0.568893 \\
\hline 250 & 1.03221 & 0.266193 \\
\hline 260 & 0.26608 & 0.068617 \\
\hline 270 & $5.44 \times 10^{-6}$ & $1.86 \mathrm{E}-07$ \\
\hline 280 & 0.26608 & 0.068617 \\
\hline 290 & 1.03221 & 0.266193 \\
\hline 300 & 2.20599 & 0.568897 \\
\hline 310 & 3.645847 & 0.940213 \\
\hline 320 & 5.178107 & 1.335363 \\
\hline 330 & 6.617963 & 1.706683 \\
\hline 340 & 7.791743 & 2.009387 \\
\hline 350 & 8.557873 & 2.206963 \\
\hline 360 & 8.823947 & 2.27558 \\
\hline
\end{tabular}


Table S12: Anisotropic dependence of hole/electron mobility for TH.

\begin{tabular}{|c|c|c|}
\hline $\begin{array}{c}\text { Angle } \\
\text { (degree) }\end{array}$ & $\begin{array}{l}\text { Hole mobility } \\
\left(\mathrm{cm}^{2} \mathrm{~V}^{-1} \mathrm{~s}^{-1}\right)\end{array}$ & $\begin{array}{c}\text { Electron mobility } \\
\left(\mathrm{cm}^{2} V^{-1} s^{-1}\right)\end{array}$ \\
\hline 0 & 5.317173 & 8.109563 \\
\hline 10 & 5.15684 & 7.865033 \\
\hline 20 & 4.695183 & 7.160927 \\
\hline 30 & 3.98788 & 6.082173 \\
\hline 40 & 3.120247 & 4.75889 \\
\hline 50 & 2.19693 & 3.35068 \\
\hline 60 & 1.329293 & 2.027393 \\
\hline 70 & 0.621993 & 0.948643 \\
\hline 80 & 0.160333 & 0.244537 \\
\hline 90 & $6.53 \mathrm{E}-07$ & $4.88 \mathrm{E}-06$ \\
\hline 100 & 0.160333 & 0.244537 \\
\hline 110 & 0.621993 & 0.948643 \\
\hline 120 & 1.329293 & 2.027397 \\
\hline 130 & 2.19693 & 3.35068 \\
\hline 140 & 3.120247 & 4.75889 \\
\hline 150 & 3.98788 & 6.082177 \\
\hline 160 & 4.695183 & 7.160927 \\
\hline 170 & 5.156843 & 7.865033 \\
\hline 180 & 5.317173 & 8.109563 \\
\hline 190 & 5.15684 & 7.865033 \\
\hline 200 & 4.695183 & 7.160927 \\
\hline 210 & 3.98788 & 6.082173 \\
\hline 220 & 3.120247 & 4.75889 \\
\hline 230 & 2.19693 & 3.35068 \\
\hline 240 & 1.329293 & 2.027393 \\
\hline 250 & 0.621993 & 0.948643 \\
\hline 260 & 0.160333 & 0.244537 \\
\hline 270 & $6.53 \mathrm{E}-07$ & $4.88 \mathrm{E}-06$ \\
\hline 280 & 0.160333 & 0.244537 \\
\hline 290 & 0.621993 & 0.948643 \\
\hline 300 & 1.329293 & 2.027397 \\
\hline 310 & 2.19693 & 3.35068 \\
\hline 320 & 3.120247 & 4.75889 \\
\hline
\end{tabular}


330

340

350

360
3.98788

4.695183

5.156843

5.317173
6.082177

7.160927

7.865033

8.109563 


\section{Section C: Figures}

a)

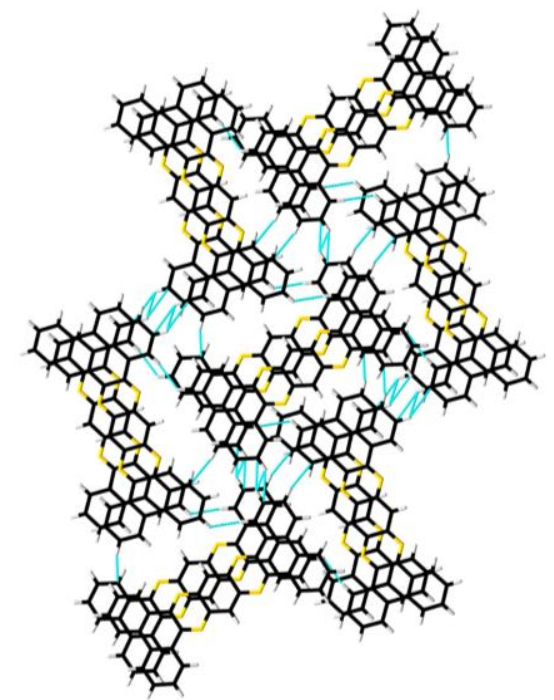

c)

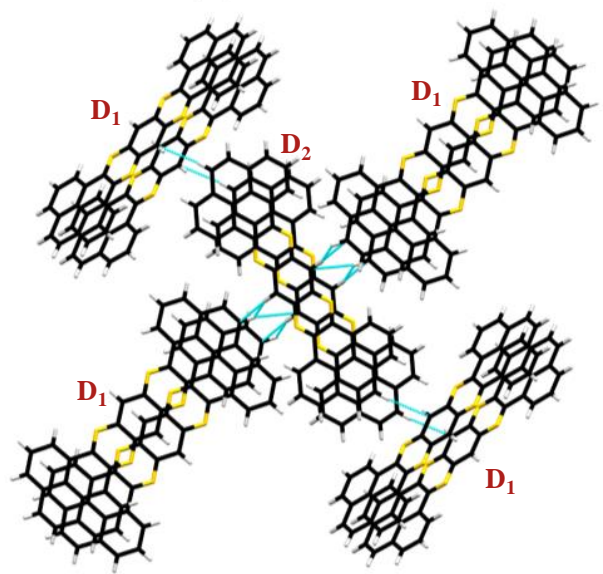

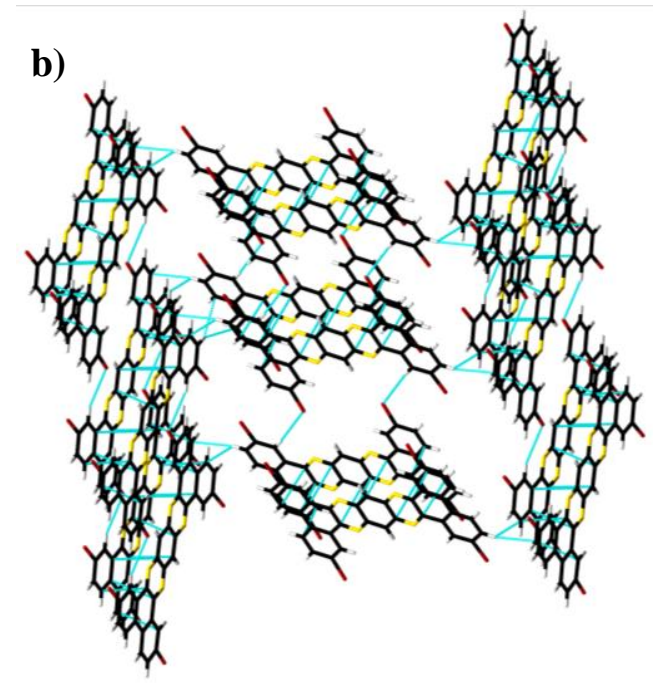

d)

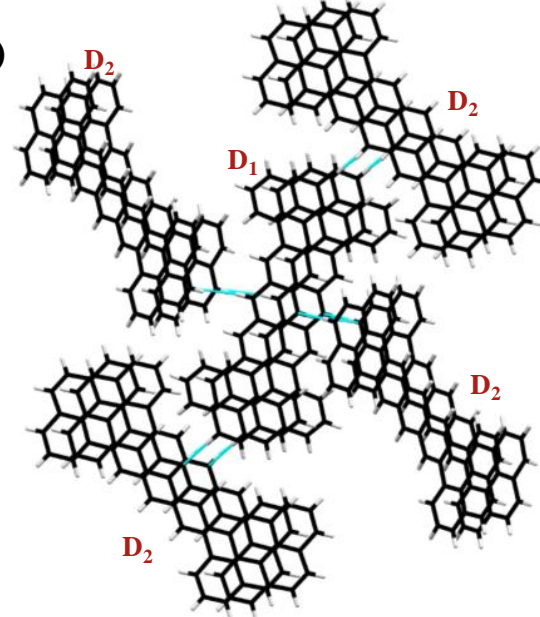

Figure S1 XRD diagram showing variation in the modes of interaction for a) NTP, b) BNTP, c) NTH and TH that has directed the crystalline packing arrangement.

a)
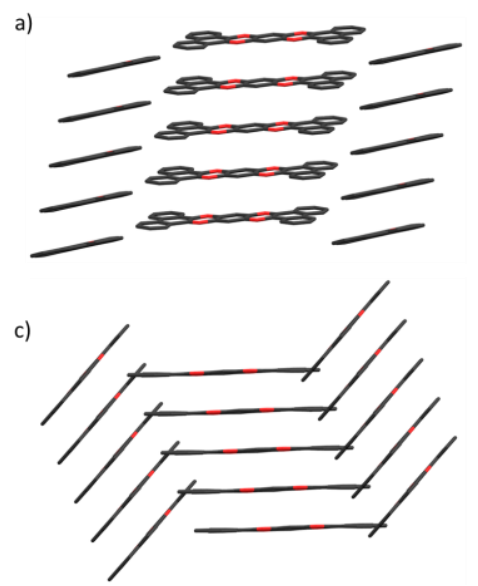

b)
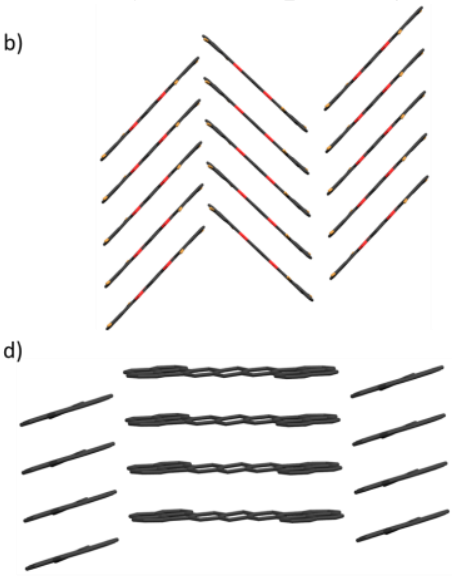

Figure S2 Crystal packing of a) NTP, b) BNTP, c) NTH and d) TH 

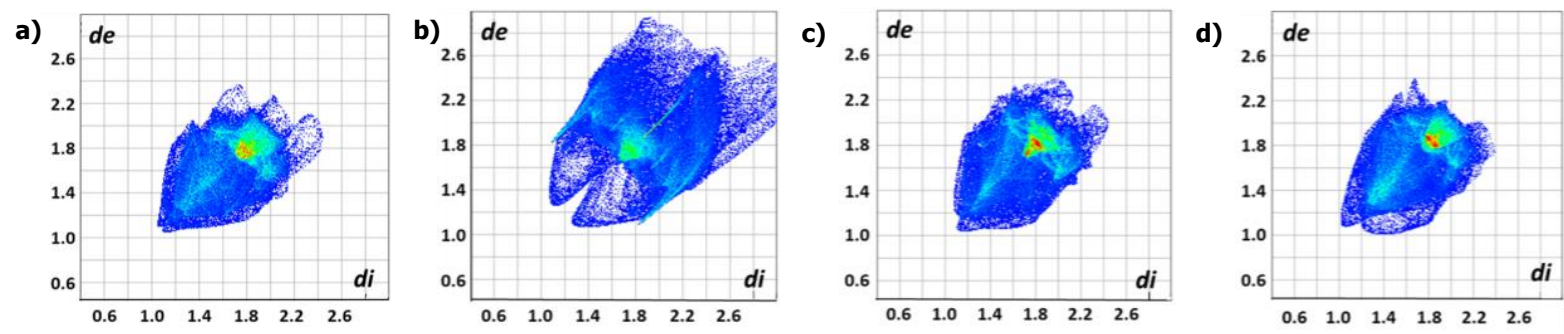

Figure S3 Hirshfeld fingerprint plots of a) NTP, b) BNTP, c) NTH and d) TH.

a)

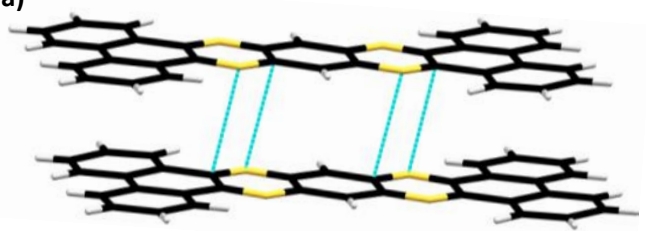

b)

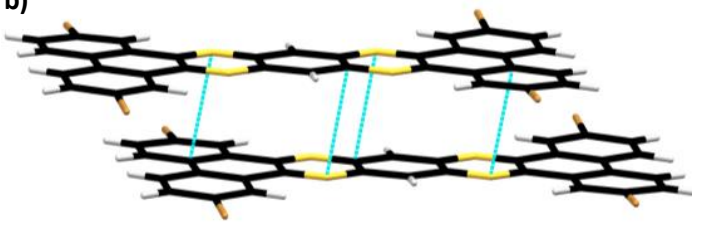

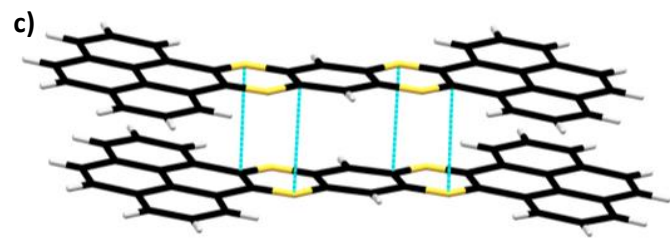

Figure S4 N $\cdots$ C interactions present in a) NTP, b) BNTP and c) NTH crystalline dimers.

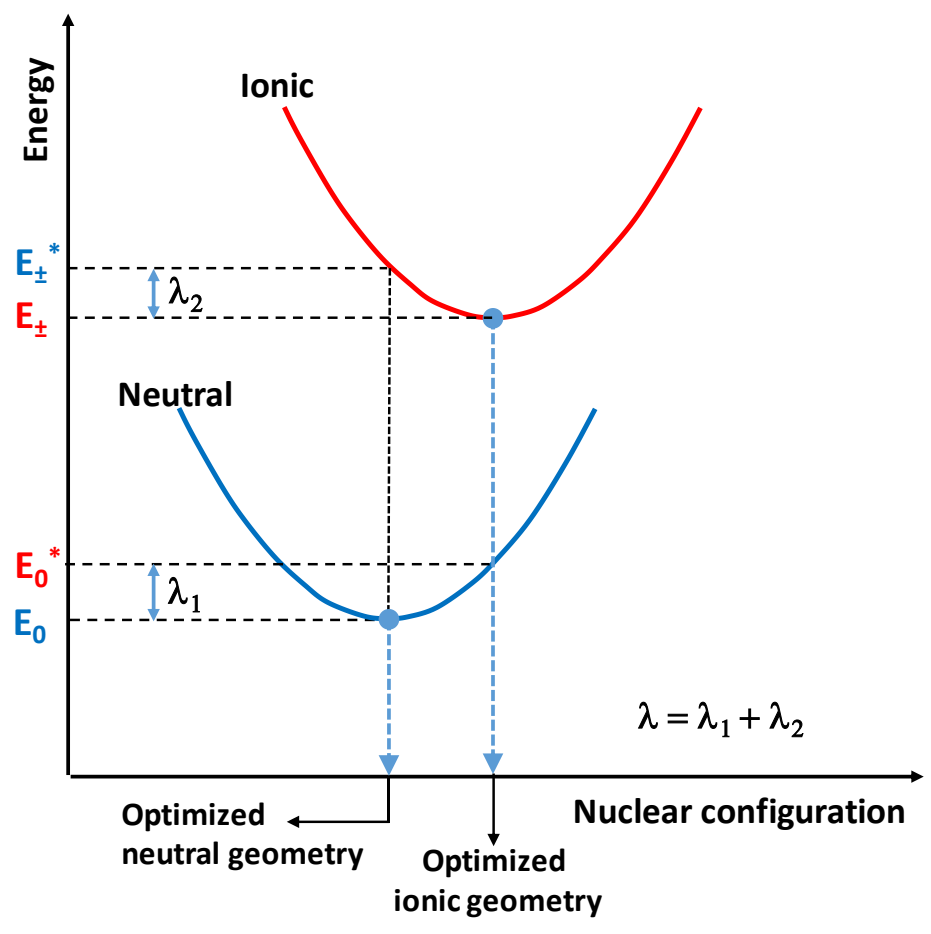

Figure S5 Schematic representation of the calculation of reorganization energy. 
a)

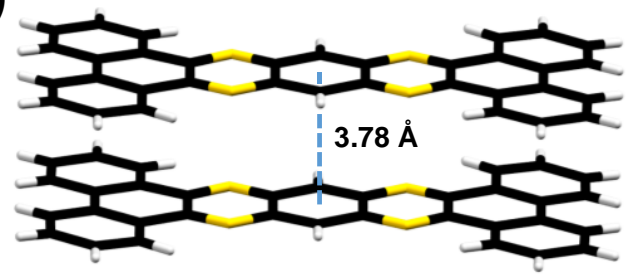

b)

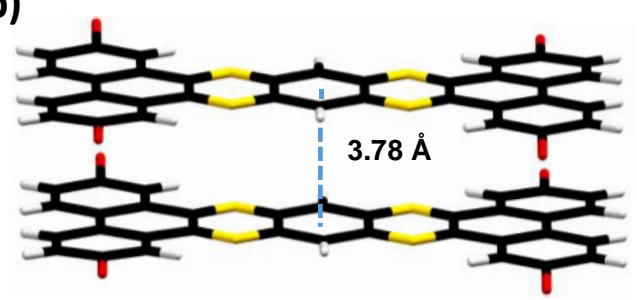

d)

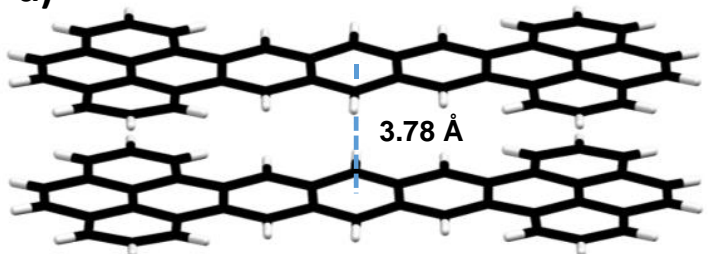

Figure S6 Shows the cofacial dimers $\left(\mathrm{D}_{0}\right)$ of the TBA derivatives at an interplanar spacing of $3.78 \AA$ a) NTP, b) BNTP, c) NTH and d) TH.

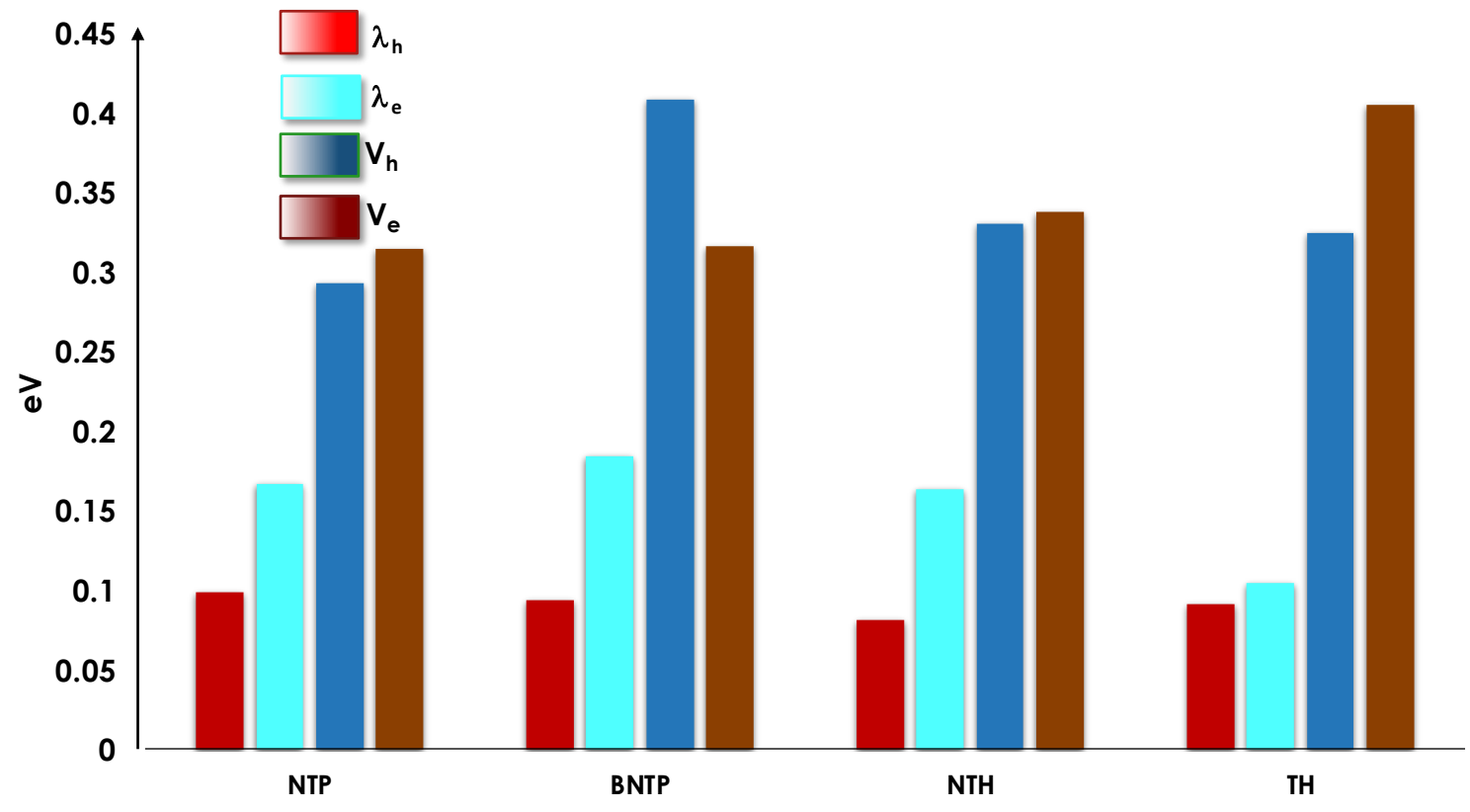

Figure S7 Charge transfer parameters of cofacial dimers $\left(D_{0}\right)$ at an interplanar spacing of $3.78 \AA$. $\lambda_{h}$ and $\lambda_{\mathrm{e}}$ are hole and electron reorganization energies while $V_{h}$ and $V_{e}$ are hole and electron coupling values respectively. 


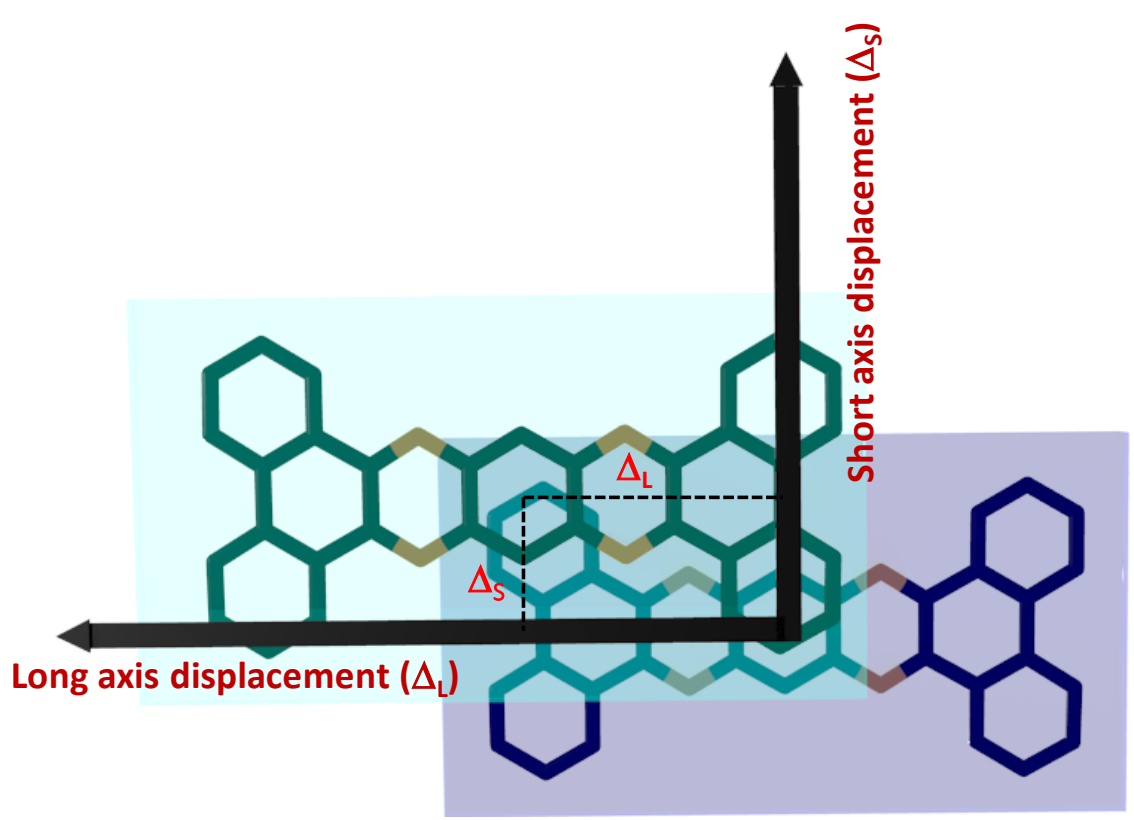

Figure S8 Representative figure showing the long $\left(\Delta_{\mathrm{L}}\right)$ and short axis $\left(\Delta_{\mathrm{S}}\right)$ displacements in NTP dimer.

a)

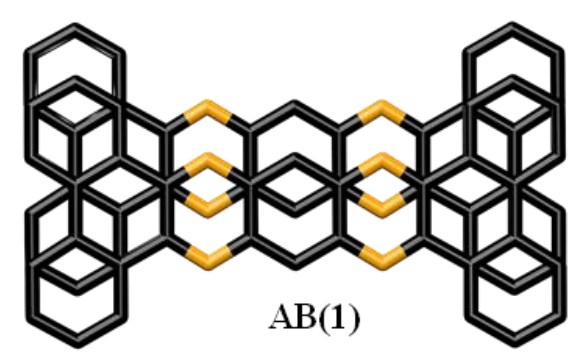

c)

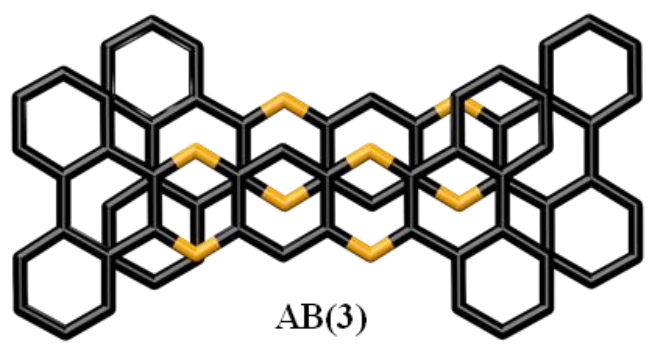

e)

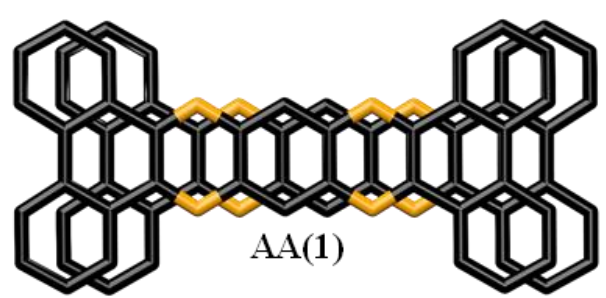

b)

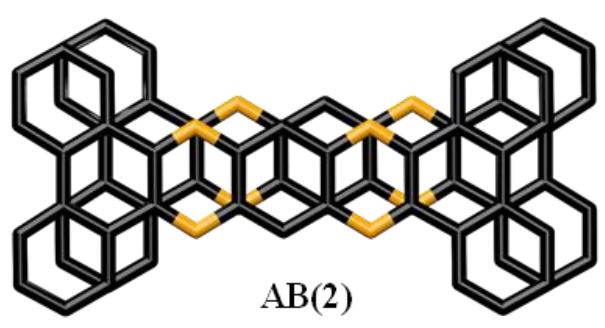

d)

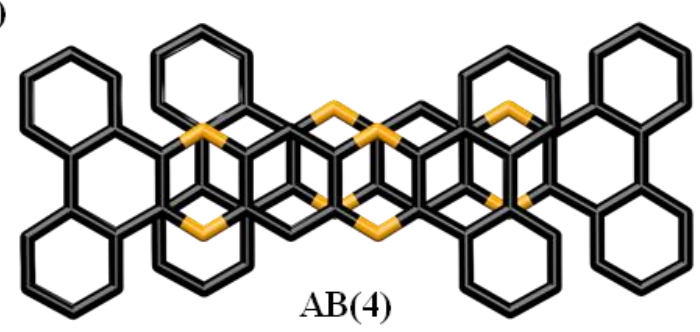

f)

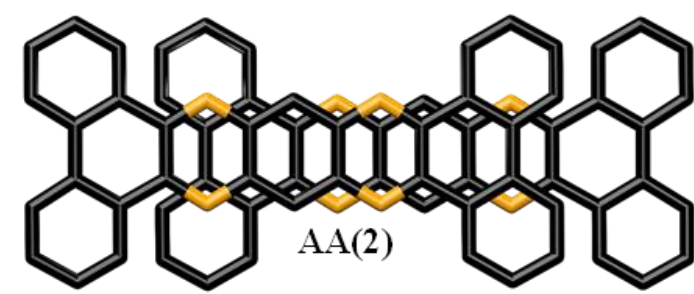

Figure S9 Graphitic $A A^{\prime}$ and $A B$ orientations of representative NTP dimer. 


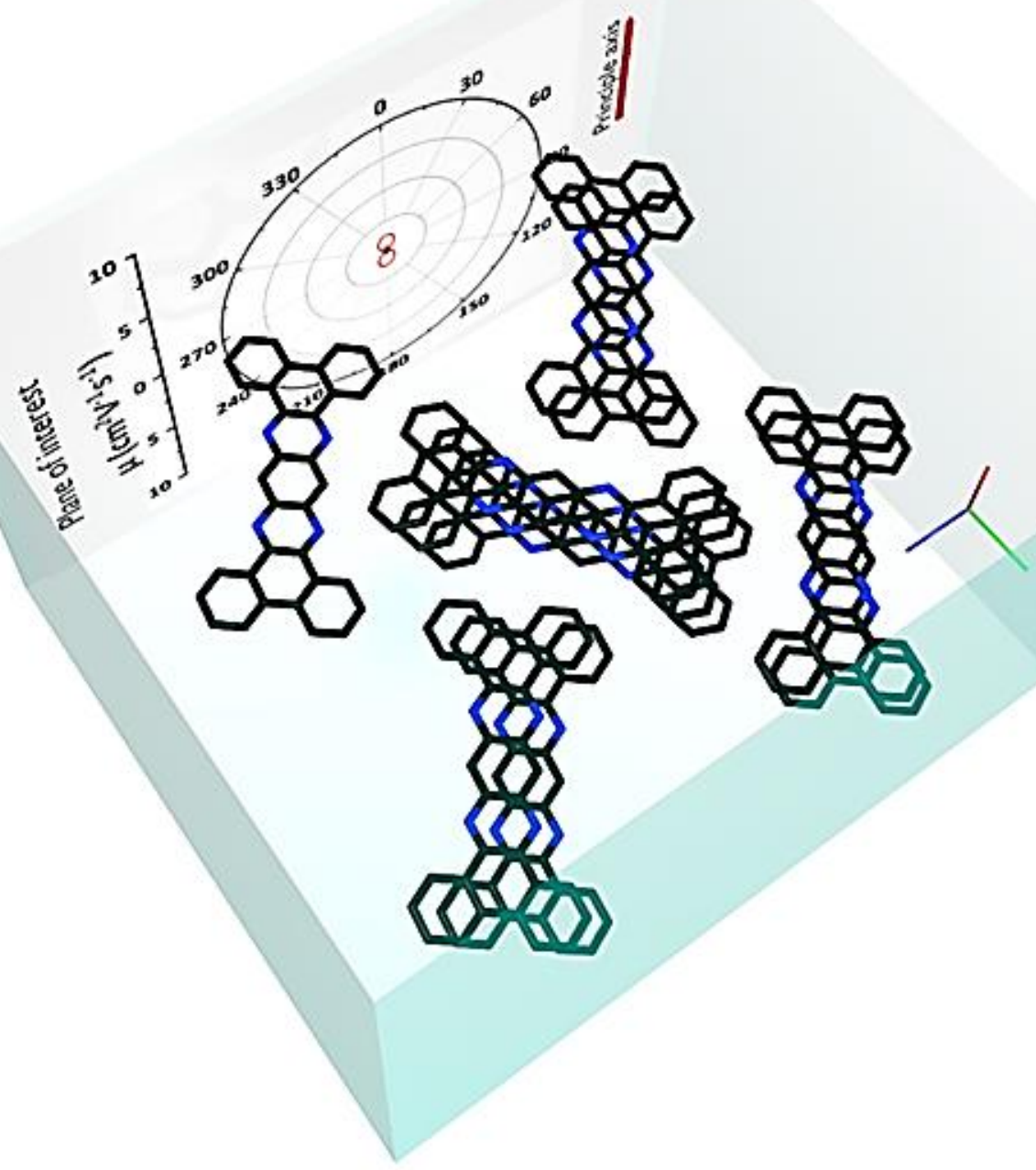

Figure S10 Representative figure showing the principle axis a and plane of interest ab for the anisotropic calculations in NTP cluster. 


\section{Section D: References}

(1) Spackman, M. A.; McKinnon, J. J. Fingerprinting Intermolecular Interactions in Molecular Crystals. CrystEngComm 2002, 4, 378-392.

(2) Spackman, M. A.; Jayatilaka, D. Hirshfeld Surface Analysis. CrystEngComm 2009, 11, 19-32.

(3) McKinnon, J. J.; Spackman, M. A.; Mitchell, A. S. Novel Tools for Visualizing and Exploring Intermolecular Interactions in Molecular Crystals. Acta Crystallogr. Sect. B Struct. Sci. 2004, $60,627-668$.

(4) Bochevarov, A. D.; Harder, E.; Hughes, T. F.; Greenwood, J. R.; Braden, D. A.; Philipp, D. M.; Rinaldo, D.; Halls, M. D.; Zhang, J.; Friesner, R. A. Jaguar: A High-Performance Quantum Chemistry Software Program with Strengths in Life and Materials Sciences. Int. J. Quantum Chem. 2013, 113, 2110-2142.

(5) Materials Science Suite 2016-2014. Schrodinger, LLC, New York, NY (USA). 2016.

(6) Deng, W.-Q.; Goddard, W. A. Predictions of Hole Mobilities in Oligoacene Organic Semiconductors from Quantum Mechanical Calculations †. J. Phys. Chem. B 2004, 108, 8614 8621.

(7) Marcus, R. A. On the Theory of Oxidation- Reduction Reactions Involving Electron Transfer. I. J. Chem. Phys. 1956, 24, 966-978.

(8) Hush, N. S. Adiabatic Rate Processes at Electrodes. I. Energy- Charge Relationships. J. Chem. Phys. 1958, 28, 962-972.

(9) Hush, N. S. Adiabatic Theory of Outer Sphere Electron-Transfer Reactions in Solution. Trans. Faraday Soc. 1961, 57, 557.

(10) Parrish, R. M.; Burns, L. A.; Smith, D. G. A.; Simmonett, A. C.; DePrince, A. E.; Hohenstein, E. G.; Bozkaya, U.; Sokolov, A. Y.; Di Remigio, R.; Richard, R. M.; et al. 〈scp >Psi4</Scp〉 1.1: An Open-Source Electronic Structure Program Emphasizing Automation, Advanced Libraries, and Interoperability. J. Chem. Theory Comput. 2017, 13, 3185-3197.

(11) Gaussian09, M. J. Frisch, G. W. T.; H. B. Schlegel, G. E. Scuseria, M. A. Robb, J. R. Cheeseman, G. S.; V. Barone, B. Mennucci, G. A. Petersson, H. Nakatsuji, M. Caricato, X. L.; H. P. Hratchian, A. F. Izmaylov, J. Bloino, G. Zheng, J. L. Sonnenberg, M.; Hada, M. Ehara, K. Toyota, R. Fukuda, J. Hasegawa, M. Ishida, T. N.; Y. Honda, O. Kitao, H. Nakai, T. Vreven, J. A. Montgomery, Jr., J. E.; Peralta, F. Ogliaro, M. Bearpark, J. J. Heyd, E. Brothers, K. N. Kudin, V. N.; Staroverov, R. Kobayashi, J. Normand, K. Raghavachari, A. Rendell, J. C.; Burant, S. S. Iyengar, J. Tomasi, M. Cossi, N. Rega, J. M. Millam, M. Klene, J. E. Knox, J. B. Cross, V. Bakken, C. Adamo, J. Jaramillo, R. G.; R. E. Stratmann, O. Yazyev, A. J. Austin, R. Cammi, C. Pomelli, J. W. O.; et al. Gaussian, Inc., Wallingford CT. 2009. 